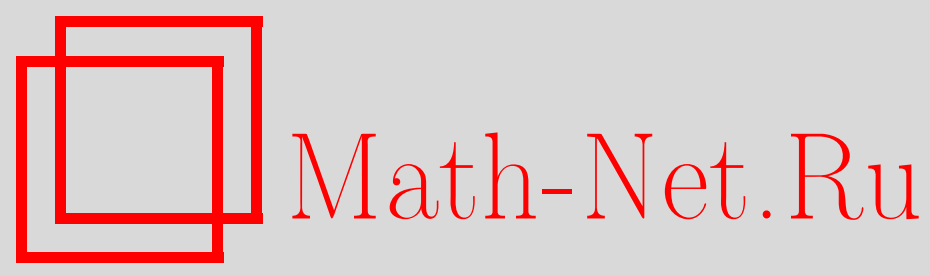

Е. В. Кашинцев, О некоторых условиях вложимости полугрупп в группы, Матем. заметки, 2001, том 70, выпуск 5, 705-717

DOI: https://doi.org/10.4213/mzm782

Использование Общероссийского математического портала Math-Net.Ru подразумевает, что вы прочитали и согласны с пользовательским соглашением http://www . mathnet.ru/rus/agreement

Параметры загрузки:

IP : 54.205 .225 .156

26 апреля 2023 г., $17: 52: 31$

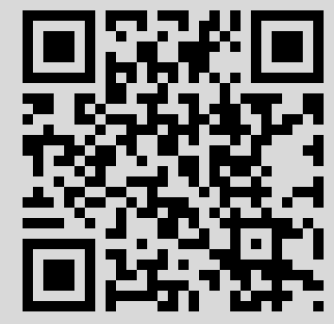




\title{
О НЕКОТОРЫХ УСЛОВИЯХ ВЛОЖИМОСТИ ПОЛУГРУПП В ГРУППЫ
}

\author{
Е. В. Кашинцев
}

В статье найдены два новых класса полугрупп, вложимых в группу, содержащих некоторые известные ранее классы полугрупп с этим свойством (теоремы 1 и 3 ). Кроме того, построены примеры не вложимых в группу полугрупп из класса $K_{p, r}^{2}$ при $p+r \geqslant 6$ (теорема 2 ), что опровергает известное ранее предположение.

Библиографояи: 9 названий.

Пусть

$$
S=\left\langle\left\langle a_{1}, a_{2}, \ldots, a_{n} ; A_{i}=B_{i}, i \in I\right\rangle\right\rangle
$$

- полугруппа, заданная образующими $a_{1}, a_{2}, \ldots, a_{n}$ и определяющими соотношениями $A_{i}=B_{i}(i \in I)$. Мы будем всегда предполагать определяющие слова $A_{i}$ и $B_{i}$ непустьми и попарно различньми. Символы $\equiv, \stackrel{s}{=}, \stackrel{g}{=}$ используются соответственно для обозначения графического равенства слов, равенства слов в полугрупше $S$ и равенства слов в группе $G$ с заданием

$$
G=\left\langle a_{1}, a_{2}, \ldots, a_{n} ; A_{i} B_{i}^{-1}=1, i \in I\right\rangle .
$$

Слово в образующих $a_{1}, a_{2}, \ldots, a_{n}$ без отрищательных степеней, включая пустое, будем называть позитивным. Слово, обратное (в свободной группе) позитивному слову, назьваем негативным.

Назовем $S$-куском относительно (1) такое слово $X$, для которого при некоторых $U_{1}$, $U_{2}, V_{1}, V_{2}$ слова $U_{1} X U_{2}, V_{1} X V_{2}$ являются определяющими, причем либо $U_{1}$ и $V_{1}$, либо $U_{2}$ и $V_{2}$ графически не совпадают.

Условие $C_{s}(p)$ для (1) состоит в том, что ни одно из определяющих слов не может быть представлено произведением менее чем $p S$-кусков.

Условие $D(q)$ для (1) состоит в том, что ни в левом, ни в правом графе нет циклов (т.е. замкнутых путей) с числом ребер менее $q$.

Заметим, что $C_{s}(1)$ и $D(1)$ указьвают на отсутствие соответствующих ограничений; $C_{s}(2)$ означает, что определяющее слово не является собственной частью другого определяющего слова; $D(2)$ предполагает несократимость каждого определяющего соотношения $A_{i}=B_{i}$, т.е. для каждого $i \in I$ слова $A_{i}$ и $B_{i}$ начинаются различными буквами.

Следуя Адяну [1], [2], введем понятие левого и правого графа для задания (1). Вершинами как левого, так и правого графа для (1) являются буквы алфавита $a_{1}, a_{2}, \ldots, a_{n}$. Каждому определяющему соотношению $A_{i}=B_{i}$ соответствует ребро левого графа, 
соединяющее начальные буквы слов $A_{i}$ и $B_{i}$, и ребро правого графа, соединяющее конечные буквы этих слов.

В работе [3] изучаются классы $K_{p}^{q}$. По определению полугрупша $S$ принадлежит классу $K_{p}^{q}$, если она обладает заданием, удовлетворяюшим условиям $C_{s}(p)$ и $D(q)$.

Напомним некоторые результаты. Адяном [1], [2] показано, что все полугруппы из класса $K_{1}^{\infty}=\bigcap_{q} K_{1}^{q}$ вложимы в группы. Мальцевьм в [4] был построен пример полугруппы, принадлежащей классу $K_{2}^{2}$, не вложимой в групу. Более простой пример указан в [2]. Такова полугруппа $S=\langle\langle a, b, c, d ; a b=e d$, aed $=c e d\rangle\rangle$.

Один из основньг результатов работы [3] утверждает, что полугрупшы из классов $K_{4}^{2}$ и $K_{3}^{3}$ вложимы в группы. Помимо этого, в [3] для любого $q>2$ построены примеры полугрупп из класса $K_{2}^{q}$, не вложимых в групшы. В этих примерах полугрупп не выполнялось сокращение слева. Оставались открытыми вопросы о вложимости в группы полугрупп из класса $K_{3}^{2}$ и о существовании примеров полугрупп с двусторонним сокращением, принадлежащих классам $K_{2}^{q}$ при $q>2$, не вложимьх в группы. Положительньй ответ на оба данньх вопроса дал Губа [5]. Как показьвает пример полугруппы $\langle\langle a, b, c, d, x, y ; a x c=b y d, a y c=b x d\rangle\rangle$, приведенный в [5], включение $K_{4}^{2} \cup K_{3}^{3} \subset K_{3}^{2}$ является собственньм.

В недавней работе [6] рассматривается другой класс полугрупп, также включающий классы $K_{4}^{2}$ и $K_{3}^{2}$ и содержащий примеры полугрупп, не входящих в $K_{3}^{2}$. Этот класс полугрупп определяется так называемьм условием $\Omega$ и условием $C(6)$, налагаемьми на группу $G$ с заданием (2).

Цель настоящей работы - указать новые классы полугрупп, вложимых в группы.

Пусть

$$
G=\left\langle a_{1}, \ldots, a_{n} ; R_{j}=1, j \in J\right\rangle
$$

- задание групшы $G$, где множество $\left\{R_{j} \mid j \in J\right\}$ симметризовано, т.е. состоит из всевозможных циклических перестановок слов $A_{i} B_{i}^{-1}$ из (2) и им обратньх. Заметим, что из вьполнения условия $D(2)$ для $(1)$ следует циклическая несократимость каждого слова $R_{j}$.

Напомним, что слово $X$ назьвается куском относительно (3), если

$$
R_{i} \equiv X U, \quad R_{j} \equiv X V \quad \text { и } R_{i} \not \equiv R_{j}
$$

для некоторьх $i, j, U$ и $V$.

Условие $C(p)$ для (3) состоит в том, что никакое определяющее слово $R_{j}$ не является произведением менее чем $p$ кусков.

ОПРЕДЕЛЕНИЕ 1. Условие $\Omega$ для (3) состоит в том, что никакое подслово вида

$$
X^{-1} A_{i} Y^{-1} \text { или } X^{-1} B_{i} Y^{-1}
$$

определяющего слова $R_{j}$, где $X, Y$ - позитивные слова, хотя бы одно из которых непусто, не может быть представлено произведением менее чем четырех кусков.

Основной результат работы [6] утверждает, что если группа $G$ с заданием (3) удовлетворяет условиям $C(6)$ и $\Omega$, то полугрупа $S$ с заданием (1) вложима в группу. 
Лемма 1 [3, лемма 3.2]. Позитивное слово $X$ или негативное слово $X^{-1}$ тогда и только тогда является куском, когда $X$ является $S$-куском.

Через $|W|_{q}$ (соответственно $\left.|W|_{s}\right)$ обозначим минимальное число кусков $(S$-кусков), в произведение которьх можно разложить слово $W$. Если такое разложение невозможно, то полагаем $|W|_{g}=\infty$ (соответственно $\left.|W|_{s}=\infty\right)$.

ЛЕмма 2 [3, лемма 3.3]. Если $W$ - позитивное слово, то $|W|_{s}=\left|W^{\varepsilon}\right|_{g}(\varepsilon= \pm 1)$, включая случай, когда $|W|_{s}=\infty$.

ЛЕмма 3. Если задание (3) группы $G$ удовлетворяет условию $\Omega$, то (3) удовлетворяет также условию $C(6)$.

ДокАЗАТЕЛЬСТво. Пусть $R$ - определяющее словогруппы $G$. Если $R$ не разложимо в произведение кусков, то для $R$ условие $C(6)$ вьполнено по определению.

Пусть $R$ разложимо в произведение кусков. Тогда каждая его буква является куском (подслово куска является куском). Пусть $R \equiv B_{1}^{-1} A B_{2}^{-1}$ и $B_{1} B_{2} \equiv B$. Пусть $b$-первая буква слова $B$. Тогда в силу условия $\Omega$ слово $b^{-1} A$ не разложимо в произведение менее четьрех кусков. Отсюда $|A|_{g} \geqslant 3$. Симметрично доказьвается, что $|B|_{g} \geqslant 3$.

Допустим, $R \equiv X_{1} X_{2} X_{3} X_{4} X_{5}$, где каждое $X_{i}$ является куском. Заметим, что слово $A$ не может содержать более трех кусков $X_{i}$ целиком, так как иначе на долю $B^{-1}$ (a значит, и $B$ ) приходилось бы не более двух кусков. Предположим, $A \equiv X_{i}^{\prime \prime} X_{i+1} X_{i+2}^{\prime}$, где $X_{i} \equiv X_{i}^{\prime} X_{i}^{\prime \prime}, X_{i+2} \equiv X_{i+2}^{\prime} X_{i+2}^{\prime \prime}$ и хотя бы одно из слов $X_{i}^{\prime}$ или $X_{i+2}^{\prime \prime}$ было непусто. В силу условия $\Omega$ имеем $\left|X_{i}^{\prime} A\right|_{g} \geqslant 4$ или соответственно $\left|A X_{i+2}^{\prime \prime}\right| g \geqslant 4$. С другой стороны, $\left|X_{i} X_{i+1} X_{i+2}^{\prime}\right|_{g} \leqslant 3$ и $\left|X_{i}^{\prime \prime} X_{i+1} X_{i+2}\right|_{g} \leqslant 3$. Противоречие. Если слово $A$ содержит целиком два куска $X_{i}$, то рассуждения симметричны с заменой $A$ на $B$. Мы доказали, что $R$ не разложимо в произведение пяти кусков. Тем более невозможно разложение $R$ в произведение менее пяти кусков. Следовательно, $|R|_{g} \geqslant 6$.

Лемма 3 позволяет переформулировать основной результат работы [6] следующим образом: если группа $G$ с заданием (3) удовлетворяет условию $\Omega$, то полугруппа $S$ с заданием (1) вложима в әруппу.

Будем говорить, что полугруппа $S$ с заданием (1) принадлежит классу $\left(K_{1}^{2}\right)^{\Omega}$, если для $S$ выполнено $D(2)$ и соответствующее задание (3) группы $G$ удовлетворяет условию $\Omega$.

Слово $A$ назовем изолированным в определяющем соотношении $A=B$ полугруппы $S$, если первая и последняя буквы слова $B$ не являются $S$-кусками. Поскольку по предположению все определяюшие слова полугруппы $S$ попарно различны, то каждое определяющее слово $A$ входит в единственное определяющее соотношение $A=B$ (или $B=A$ ), поэтому можно говорить об изолированности слова $A$ относительно (1).

ОПРЕДЕЛЕНИЕ 2. Условие $\Phi$ для (1) состоит в том, что если определяющее слово $A$ разложимо в произведение менее чем трех кусков, то $A$ изолировано относительно (1). Класс полугрупп $S$ с заданием (1), удовлетворяющих условиям $\Phi$ и $D(2)$, обозначим через $\left(K_{1}^{2}\right)^{\Phi}$.

Покажем, что

$$
K_{3}^{2} \cup\left(K_{1}^{2}\right)^{\Omega} \subset\left(K_{1}^{2}\right)^{\Phi}
$$


Действительно, тот факт, что выполнение условия $C_{s}(3)$ влечет выполнение условия $\Phi$, непосредственно следует из определения этих условий.

Пусть для (3) вьполняется $\Omega$, и пусть $A=B$ - определяющее соотношениеполугруппы $S$. Пусть $B \equiv b B_{1} c$. Если $b^{-1}$ и $c^{-1}$ не являются кусками, то по лемме 1 буквы $b$ и $c$ не являются $S$-кусками. Тогда слово $A$ является изолированным относительно $(1)$ и для $A$ условие $\Phi$ вьполнено. Пусть $b^{-1}$ является куском. Тогда в силу $\Omega$ слово $b^{-1} A$ не разложимо в произведение менее четырех кусков и $A$ не разложимо в произведение менее трех кусков. Поскольку $A$ - позитивное слово, то $|A|_{g}=|A|_{s}$ по лемме 2. Отсюда следует выполнение $C_{s}(3)$ для $A$, а значит, и условия $\Phi$ для $A$. Аналогично рассматривается случай, когда $c^{-1}$ является куском.

Пример полугрупшы

$$
S_{1}=\langle\langle a, b, x, y, z, t ; x a y=y a x, x b y=y b x, a=z a t\rangle\rangle
$$

показывает, что включение (4) является собственным.

Теорема 1. Каждая полугруппа $S$ из класса $\left(K_{1}^{2}\right)^{\Phi}$ вложима в группу.

Доказательство теоремы опирается на технику диаграмм Линдона-Ван Кампена [7, гл. 5]. Учитывая особенности задания (3) группы $G$, доказывается усиленный вариант (лемма 5) формулы кривизны [7, гл. 5, теорема 4.3] для $(4,4)$-карт.

Пусть полугруппа $S$ с заданием (1) принадлежит классу $\left(K_{1}^{2}\right)^{\Phi}$. Перепишем (1) в виде

$$
S=\left\langle\left\langle a_{1}, \ldots, a_{n} ; A_{l}=B_{l}, A_{k}=B_{k}(l \in L, k \in K)\right\rangle,\right.
$$

где $L \cup K=I, L \cap K=\varnothing$ и множество $\left\{A_{k}=B_{k}, k \in K\right\}$ состоит из тех и только тех определяющих соотношений из (1), которые содержат изолированное слово.

Добавим к (5) образующие $x_{l}, l \in L$, и заменим каждое соотношение $A_{l}=B_{l}$ парой новьх соотношений $x_{l}=A_{l}$ и $x_{l}=B_{l}$. Получим новое задание полугрупшы $S$ :

$$
S=\left\langle\left\langle a_{1}, \ldots, a_{n}, x_{1}, x_{2}, \ldots ; x_{l}=A_{l}, x_{l}=B_{l}, A_{k}=B_{k}(l \in L, k \in K)\right\rangle\right\rangle .
$$

Рассмотрим соответствующую группу $G$ :

$$
G=\left\langle a_{1}, \ldots, a_{n}, x_{1}, x_{2}, \ldots ; A_{l} x_{l}^{-1}=1, B_{l} x_{l}^{-1}=1, A_{k} B_{k}^{-1}=1(l \in L, k \in K)\right\rangle .
$$

Пусть

$$
G=\left\langle a_{1}, \ldots, a_{n}, x_{1}, x_{2}, \ldots ; P_{l^{\prime}}=1, Q_{k^{\prime}}=1\left(l^{\prime} \in L^{\prime}, k^{\prime} \in K^{\prime}\right)\right\rangle,
$$

где $\left\{P_{l^{\prime}}\right\}$ и $\left\{Q_{k^{\prime}}\right\}$ получены замыканием соответствуюших множеств $\left\{A_{l} x_{l}^{-1}, B_{l} x_{l}^{-1}\right\}$ и $\left\{A_{k} B_{k}^{-1}\right\}$ относительно операций циклического сдвига и взятия обратного слова. Элементы множеств $\left\{P_{l^{\prime}}\right\}$ и $\left\{Q_{k^{\prime}}\right\}$ будем для краткости назьвать соответственно $P$-словами и $Q$-словами.

Условие $T(q)$ для группы $G$ с симметризованным множеством определяющих слов состоит в том, что для любой последовательности определяющих слов $R_{1}, R_{2}, \ldots, R_{t}$, где $t<q$ и пары соседних слов, а также слова $R_{t}$ и $R_{1}$ не являются взаимно обратными, по меньшей мере одно из произведений $R_{1} R_{2}, \ldots, R_{t-1} R_{t}, R_{t} R_{1}$ не допускает сокращений. 
ЛЕмма 4. Если полугруппа $S$ принадлежит классу $\left(K_{1}^{2}\right)^{\Phi}$, то задание (8) группь $G$ удовлетворяет условиям $C(4)$ и $T(4)$.

ДоКАЗАТЕЛЬСТво. Вьполнимость $C(4)$ для (8) непосредственно вытекает из следующих фактов. Всякий кусок является позитивньм или негативньм словом. Если кусок $X$ содержит $x_{i}^{\varepsilon}(\varepsilon= \pm 1)$, то $X \equiv x_{i}^{\varepsilon}$. Всякое позитивное $X$ или негативное $X^{-1}$ слово в алфавите $\left\{a_{1}, a_{2}, \ldots, a_{n}\right\}$ является куском тогда и только тогда, когда $X$ является $S$-куском относительно (1).

Допустим, для (8) не вьполняется условие $T(4)$. Тогда найдутся определяющие слова $R_{1}, R_{2}, R_{3}$ такие, что каждое из произведений $R_{1} R_{2}, R_{2} R_{3}, R_{3} R_{1}$ допускает сокращение и не равно 1 в свободной групше. В этом случае некоторое $R_{i}$ имеет вид $R_{i} \equiv a^{\varepsilon} T b^{-\varepsilon}$, где $\varepsilon= \pm 1$ и $a, b-$ буквы, являющиеся кусками. Можно считать $i=1$. Тогда из вида соотношений (7) следует, что $R_{1} \equiv A^{\varepsilon} B^{-\varepsilon}$, где $A=B$ - определяющее соотношение из (6). Если бы $R_{1}$ было $Q$-словом, то в силу определения задания (6) хотя бы одна из букв $a, b$ не являлась куском. Допустим, $R_{1}-P$-слово. Тогда $a \equiv x_{j}$ или $b \equiv x_{j}$ для некоторой буквы $x_{j}$. В случае $a \equiv x_{j}$ имеем $R_{3} \equiv c^{\varepsilon} U x_{j}^{-\varepsilon}, R_{2} \equiv b^{\varepsilon} V c^{-\varepsilon}$, и мы получаем противоречие, повторив рассуждение для $R_{2}$ вместо $R_{1}$. Аналогично, в случае $b \equiv x_{j}$ получаем противоречие для $R_{3}$ вместо $R_{1}$.

СлЕдСТВИЕ 1. В каждой конечно определенной полугруппе из класса $\left(K_{1}^{2}\right)^{\Phi}$ разрешима проблема равенства слов.

ДокАЗАТЕЛЬСТво следует из теоремы 1 , леммы 4 и разрешимости проблемы равенства слов в конечно определенных группах с условиями $C(4)$ и $T(4)$ (см. [7, гл. 5]).

ЗАмЕЧАнИЕ. Известна разрешимость проблемы равенства слов для полугрупп с условием $C_{s}(3)$ и даже для несколько более широких классов полугрупп (см. [8]). Однако для всех этих полугрупп характерно то, что любое слово имеет лишь конечное число равньх ему в полугруппе слов. В классе $\left(K_{1}^{2}\right)^{\Phi}$ имеются полугрупы, в которых найдутся слова, имеющие бесконечное число слов, равных им. Это связано с допущением, что одно определяющее слово может быть собственной частью другого определяющего слова.

Пусть $M$ - произвольная карта. Через $V^{\bullet}, E^{\bullet}, F^{\bullet}$ будем обозначать соответственно число граничных вершин, число граничных ребер и число граничных областей карты $M$. Условимся считать, что граничный цикл связной односвязной карты $M$ и граничные циклы ее областей ориентированы в одном направлении. В отличие от [7, гл. 5], әраничной областью карты $M$ будем назьвать область $D$, граничньй цикл $\partial D$ которой имеет ребро в $\partial M$. Если все внутренние вершины $v$ карты $M$ имеют степень $d(v) \geqslant q$, а все внутренние области $D$ имеют степень $d(D) \geqslant p$, то $M$ назьвается $(q, p)$-картой; $\sum d(v), \sum d(D)$ обозначают суммирование по всем вершинам и по всем областям карты $M$. Знак $\sum^{\bullet}$ применяется для суммирования по всем граничным вершинам или граничньг областям, а знак $\sum^{\circ}$ - для суммирования по всем внутренним вершинам или внутренним областям.

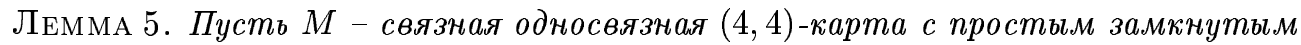
граничным контуром $\partial M$, и пусть все граничные вериины, кроме быть может двух $v_{1} u v_{2}$, имеют степень не меньие четырех, а при $i=1,2 d\left(v_{i}\right) \geqslant 3$. Тогда в $M$ найдутся две области $D_{1}$ и $D_{2}$, граничные контуры которых состоят каждый из двух ребер: граничного и внутреннего. 
ДокАЗАТЕЛЬСТво. Для произвольной карты $M$ (см. [7, гл. 5, формула 3.2]) имеет место равенство

$$
\begin{aligned}
P(Q-h)= & \sum^{\bullet}\left[\frac{p}{q}+2-d(v)\right]+\sum^{\circ}[p-d(v)] \\
& +\frac{p}{q} \sum^{\bullet}[q-d(D)]+\frac{p}{q} \sum^{\circ}[q-d(D)]+\frac{p}{q}\left(V^{\bullet}-E^{\bullet}\right),
\end{aligned}
$$

где $Q$ - число компонент карты $M, h$ - число дырок в $M, p$ и $q$ - натуральные числа, удовлетворяющие уравнению $1 / p+1 / q=1 / 2$.

В нашем случае $p=q=4, Q=1, h=0$. Так как $M$ есть $(4,4)$-карта, то $\sum^{\circ}[4-d(v)]$ и $\sum^{\circ}[4-d(D)]$ принимают неположительные значения. Поскольку $\partial M-$ простой замкнутый контур, то $V^{\bullet}=E^{\bullet}$. Отсюда как следствие получаем неравенство

$$
\sum^{\bullet}[4-d(D)]+\sum^{\bullet}[3-d(v)] \geqslant 4 .
$$

Далее, принимая во внимание условие леммы и наше определение граничной области, получаем

$$
\begin{aligned}
4 & \leqslant \sum^{\bullet}[3-d(v)]+\sum^{\bullet}[3-d(D)]+F^{\bullet} \leqslant \sum^{\bullet}[3-d(v)]+V^{\bullet}+\sum^{\bullet}[3-d(D)] \\
& =\sum^{\bullet}[4-d(v)]+\sum^{\bullet}[3-d(D)] \leqslant 2+\sum^{\bullet}[3-d(D)] .
\end{aligned}
$$

Отсюда

$$
\sum^{\bullet}[3-d(D)] \geqslant 2
$$

Из условия леммы, очевидно, следует, что $M$ имеет по крайней мере две области. В этом случае так как $\partial M-$ простой замкнутый контур, граница каждой области содержит хотя бы одно внутреннее ребро. В частности, $d(D) \geqslant 2$ для каждой граничной области $D$. Тогда из (9) следует, что найдутся две области $D_{1}$ и $D_{2} \mathrm{c} d\left(D_{i}\right)=2$.

ДОКАЗАТЕЛЬСТво ТЕОРЕМЫ 1 . Пусть $U$ и $V$-позитивные слова в алфавите $\left\{a_{1}, a_{2}\right.$, $\left.\ldots, a_{n}, x_{1}, x_{2}, \ldots\right\}$ из (6), (8). Наша задача доказать, что если $U \stackrel{g}{=} V$, то $U \stackrel{s}{=} V$.

Пусть $M$ - минимальная диаграмма над заданием (8) с меткой граничного конту-

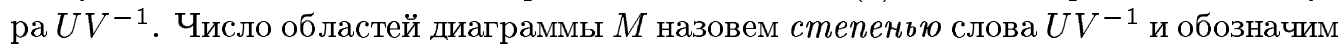
$\operatorname{deg}\left(U V^{-1}\right) ; \ell(W)$ обозначает длину слова $W$. Доказательство теоремы ведем индукцией по упорядоченным парам

$$
K(U, V)=\left(\operatorname{deg}\left(U V^{-1}\right), \ell\left(U V^{-1}\right)\right),
$$

где порядок на множестве таких пар определяется лексикографически.

Если $\operatorname{deg}\left(U V^{-1}\right)=0$, то $U \equiv V$ и теорема доказана.

Пусть $\operatorname{deg}\left(U V^{-1}\right)>0$ и слова $U$ и $V$ начинаются с одной буквы: $U \equiv a U^{\prime}, V \equiv a V^{\prime}$. Поскольку $\operatorname{deg}\left(U^{\prime} V^{\prime-1}\right)=\operatorname{deg}\left(U V^{-1}\right)$, то $K\left(U^{\prime} V^{\prime}\right)<K(U, V)$, откуда по индукции заключаем, что $U^{\prime} \stackrel{s}{=} V^{\prime}$ и, следовательно, $U \stackrel{s}{=} V$. Аналогично рассматривается случай, когда $U$ и $V$ оканчиваются на одну букву. Поэтому можно предположить, что слово $U V^{-1}$ циклически несократимо.

Если $\operatorname{deg}\left(U V^{-1}\right)=1$, то $U=V$ - определяющее соотношение полугруппы $S$. 
Предположим, что $\partial M$ имеет самопересечение. Тогда $\partial M=\alpha_{1} \beta \alpha_{2}$, где $\beta$ - непустой простой замкнутый путь. Пусть метка $W$ пути $\beta$ входит в $U$. Имеем $W \stackrel{g}{\underline{g}} 1$ и $K(W, 1)<K(U, V)$. По индуктивному предположению $W \stackrel{s}{=} 1$, что невозможно при непустых определяющих словах полугрупшы $S$. Аналогично доказьвается, что $W$ не входит в $V$. Следовательно, имеем $U \equiv U_{1} U_{2}, V \equiv V_{1} V_{2}$ и $W \equiv U_{2} V_{2}^{-1}$. Тогда для $i=1,2$ получаем $U_{i} \stackrel{g}{=} V_{i}, K\left(U_{i}, V_{i}\right)<K(U, V)$ и по индуктивному предположению $U_{i} \stackrel{s}{=} V_{i}$. Отсюда $U \stackrel{s}{=} V$.

Будем считать теперь, что $\partial M-$ простой замкнутый путь и $\operatorname{deg}\left(U V^{-1}\right)>1$.

Рассмотрим случай, когда $M$ содержит область $D$, метка граничного контура которой является $Q$-словом. С точностью до циклической перестановки любое $Q$-слово имеет вид $A B^{-1}$ или $B A^{-1}$, где $A=B$ - определяющее соотношение полугруппы $S$ и $A-$ слово, изолированное относительно (1). То есть имеем $B \equiv a B^{\prime} b$, где $a, b$ не являются $S$-кусками, а значит, не являются кусками относительно (8). Поскольку $M-$ минимальная диаграмма, то она является приведенной, поэтому буквы $a^{\varepsilon}, b^{\varepsilon}(\varepsilon= \pm 1)$ не могут входить в метки внутренних ребер. Следовательно, буквы $a, b$ входят одновременно либо в слово $U$, либо в слово $V$. Пусть, например, $U \equiv U_{1} a U_{2} b U_{3}$. Тогда $a U_{2} b \stackrel{g}{=} A$ и $U_{1} A U_{3} \stackrel{g}{=} V$ (рис. 1).

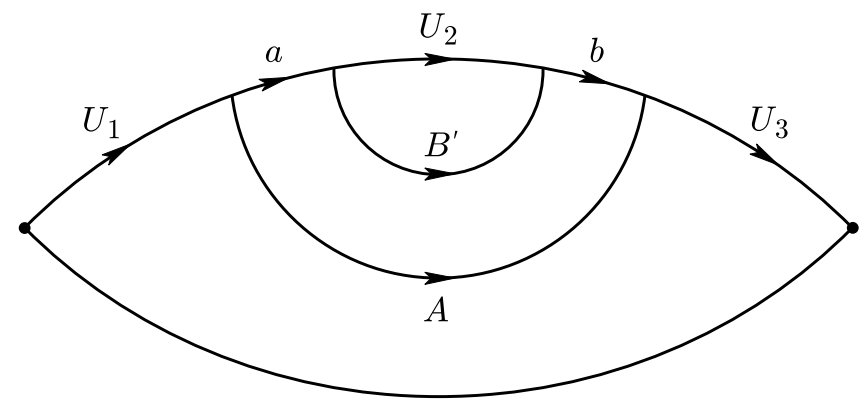

Рис. 1

Если $U_{1} A U_{3} \not \equiv V$, то $\operatorname{deg}\left(a U_{2} b A^{-1}\right)<\operatorname{deg}\left(U V^{-1}\right)$ и $\operatorname{deg}\left(U_{1} A U_{3} V^{-1}\right)<\operatorname{deg}\left(U V^{-1}\right)$. По индуктивному предположению $a U_{2} b \stackrel{s}{=} A$ и $U_{1} A U_{3} \stackrel{s}{=} V$, откуда $U \stackrel{s}{=} V$.

Если $U_{1} A U_{3} \equiv V$, то слова $U_{1}$ и $U_{3}$ пусты, поскольку $U V^{-1}$ циклически несократимо. Тогда $U \stackrel{\underline{g}}{=} B, \operatorname{deg}\left(U B^{-1}\right)<\operatorname{deg}\left(U V^{-1}\right)$ и по индуктивному предположению $U \stackrel{s}{=} B \stackrel{s}{=} A \equiv V$.

Аналогично рассматривается случай, когда буквы $a$ и $b$ входят в слово $V$. Тем самым рассмотрен случай, когда $M$ содержит область с $Q$-меткой.

Предположим теперь, что некоторая буква $x_{i}$ входит в слово $U$ или $V$. Поскольку $\partial M$ - простой замкнутый контур, то $x_{i}$ является меткой ребра граничного контура некоторой области $D$. Пусть $U \equiv U_{1} x_{i} U_{2}$, и пусть $x_{i}=B$ - соотношение, соответствующее метке $\partial D$. Тогда $U_{1} B U_{2} \stackrel{g}{=} V$, где $\operatorname{deg}\left(U_{1} B U_{2} V^{-1}\right)<\operatorname{deg}\left(U V^{-1}\right)$. По индуктивному предположению $U_{1} B_{1} U_{2} \stackrel{s}{=} V$ и вместе с соотношением $x_{i} \stackrel{s}{=} B$ получаем $U \stackrel{s}{=} V$.

Остается рассмотреть случай, когда $U$ и $V$-позитивные слова в алфавите $a_{1}, a_{2}, \ldots$, $a_{n}$ и $M$ не содержит областей с $Q$-метками. Покажем, что карта $M$ удовлетворяет условиям леммы 5 . По лемме 4 задание (8) группы $G$ удовлетворяет условиям $C(4)$ и $T(4)$. Тогда $M$ является $(4,4)$-картой (см. [7, гл. 5]). 
Заметим, что если $e_{1}, e_{2}$ - последовательные ребра, входящие в граничньй контур некоторой области диаграммы $M$, и метки ребер $e_{i}$ имеют вид $\varphi\left(e_{1}\right) \equiv X^{\varepsilon}, \varphi\left(e_{2}\right) \equiv Y^{-\varepsilon}$, где $\varepsilon= \pm 1$ и $X, Y$ - позитивные слова, то одно из слов $\varphi\left(e_{i}\right)$ содержит вхождение некоторой буквы $x_{j}^{ \pm 1}$; если при этом $e_{i}$ - внутреннее ребро, то $\varphi\left(e_{i}\right) \equiv x_{j}^{ \pm 1}$.

Пусть $\nu \tau^{-1}$ - граничный контур диаграммы $M$, где $\varphi(\nu) \equiv U$ и $\varphi(\tau) \equiv V$. Пусть $v_{1}-$ начальная и $v_{2}-$ конечная вершины путей $\nu$ и $\tau$.

Допустим, $d\left(v_{i}\right)=2$ для $i=1$ или $i=2$. Пусть $e_{1}, e_{2}$ - последовательные ребра, входящие в граничньй контур диаграммы $M$, где $v_{i}-$ конец $e_{1}$. Тогда для некоторых позитивных слов $X, Y$ имеем $\varphi\left(e_{1}\right) \equiv X^{-1}, \varphi\left(e_{2}\right) \equiv Y$ при $i=1$ и $\varphi\left(e_{1}\right) \equiv X, \varphi\left(e_{2}\right) \equiv Y^{-1}$ при $i=2$. Так как $e_{1}, e_{2}$ входят в граничньй контур некоторой области диаграммы $M$, то мы получаем противоречие с предположением, что слова $U$ и $V$ не содержат вхождений букв $x_{j}$. Следовательно, $d\left(v_{i}\right) \geqslant 3(i=1,2)$.

Можно считать также, что $d(v) \geqslant 3$ для любой граничной вершины $v$ диаграммы $M$, отличной от $v_{i}$. Действительно, если $d(v)=2$, то последовательные ребра, инцидентные вершине $v$, можно объединить в одно.

Допустим, $d(v)=3$ для некоторой такой вершины $v$. Пусть $e_{1}, e_{2}-$ последовательные ребра, входящие в граничный контур диаграммы $M$, где $v-$ конец $e_{1}$. Так как $\partial M$ не имеет самопересечений, то $v$ - начало также некоторого внутреннего ребра $e_{3}$. В силу замечания вьше $\varphi\left(e_{3}\right) \equiv x_{j}^{ \pm 1}$. Так как $e_{1} e_{3}^{-1}$ и $e_{3} e_{2}$ входят в граничные контуры некоторьх областей диаграммы $M$, метки которьх являются $P$-словами, то ни одно из слов $\varphi\left(e_{1} e_{3}^{-1}\right), \varphi\left(e_{3} e_{2}\right)$ не может быть позитивным или негативным. С другой стороны, $\varphi\left(e_{1} e_{2}\right)$ - позитивное или негативное слово, и тогда одно из слов $\varphi\left(e_{1} e_{3}^{-1}\right), \varphi\left(e_{3} e_{2}\right)$ также должно быть позитивным или негативным. Противоречие. Следовательно, $d(v) \geqslant 4$ для любой граничной вершины $v$ диаграммы $M$, отличной от $v_{i}$.

Таким образом, условия леммы 5 вьполнены. Тогда по лемме 5 в $M$ найдется область с граничньм циклом $e_{1} e_{2}$, где $e_{1}-$ граничное ребро, а $e_{2}-$ внутреннее. Так как $\varphi\left(e_{1}\right)$ входит в $U V^{-1}$, то $\varphi\left(e_{1}\right)$ не содержит вхождений букв $x_{j}^{ \pm 1}$. Следовательно, $\varphi\left(e_{2}\right) \equiv x_{j}^{ \pm 1}$. Пусть для определенности $e_{1}$ входит в $\nu$. Тогда $U \equiv U_{1} B U_{2}, \varphi\left(e_{1}\right) \equiv B, \varphi\left(e_{2}\right) \equiv x_{j}^{-1}$, где $B=x_{j}$ - определяюшее соотношение полугруппы $S$. Как и выше, по индуктивному предположению имеем $U_{1} x_{j} U_{2} \stackrel{s}{=} V$, поскольку $\operatorname{deg}\left(U_{1} x_{j} U_{2} V^{-1}\right) \leqslant \operatorname{deg}\left(U V^{-1}\right)-1$, откуда $U \equiv U_{1} B U_{2} \stackrel{s}{=} V$. Теорема 1 доказана.

Наши дальнейшие рассуждения касаются полугрупп с неравномерньп распределением кусков в определяющих соотношениях.

ОПРЕДЕЛЕНИЕ 3. Условие $C_{s}(p, r)$ для (1) состоит в том, что для любого определяющего соотношения $A_{i}=B_{i}$ слово $A_{i}$ (или $B_{i}$ ) не может быть представлено произведением менее чем $p$ кусков, а $B_{i}$ (соответственно $A_{i}$ ) - менее чем $r$ кусков. По определению полугруппа $S$ принадлежит классу $K_{p, r}^{q}$, если она обладает заданием, удовлетворяющим условиям $C_{s}(p, r)$ и $D(q)$.

Заметим, что если $l=\min \{p, r\}$, то $K_{l}^{q} \supseteq K_{p, r}^{q}$. Если $p=r$, то $K_{p, r}^{q}=K_{p}^{q}$. У автора и В.С. Губы было предположение, что все полугрупшы из класса $K_{p, r}^{2}$ при $p+r \geqslant 6$ вложимы в группы. Следующая теорема опровергает это предположение.

ТЕОремА 2. Для любых $p \geqslant 3 u q \geqslant 2$ в классе $K_{2, p}^{q}$ существует конечно определенная полугруппа $S$, не вложимая в әруппу. 
ДокАЗАТЕЛЬСТво. Будем строить полугруппу $S$ из класса $K_{p, r}^{q}$, в которой не выполняется левое сокращение.

В качестве образующих полугруппы $S$ возьмем следующее множество букв, где $t_{1}=p, t_{i+1}=t_{i}(p-1)+3-p(i=1,2, \ldots, q-2)$ :

$$
\begin{gathered}
a_{0}, a_{1}, \ldots, a_{t_{1}}, \\
b_{0}, b_{1}, \ldots, b_{t_{2}}, \\
\ldots \ldots \ldots, \ldots . . \\
f_{0}, f_{1}, \ldots, f_{t_{q-3}}, \\
g_{0}, g_{1}, \ldots, g_{t_{q-2}}, \\
h_{0}, h_{1}, \ldots, h_{t_{q-1}}, \\
x_{0}, x_{1}, \ldots, x_{t_{q-1}} .
\end{gathered}
$$

В качестве определяющих для $S$ возьмем следующую систему равенств $\sigma=\bigcup_{i=1}^{q} \sigma_{i}$, где $s_{i}=t_{i+1}-(p-2)$ :

$$
\begin{aligned}
& \sigma_{1}: a_{1} a_{2} \cdots a_{t_{1}}=h_{0} x_{0}, \quad \sigma_{2}:\left\{\begin{array}{r}
b_{1} b_{2} \cdots b_{p}=a_{1} a_{0} \\
a_{0} b_{p+1} \cdots b_{2 p-1}=a_{2} a_{0} \\
\ldots \ldots \ldots \cdots \cdots \\
a_{0} b_{s_{1}} \cdots b_{t_{2}}=a_{t_{1}} a_{0}
\end{array}\right.
\end{aligned}
$$

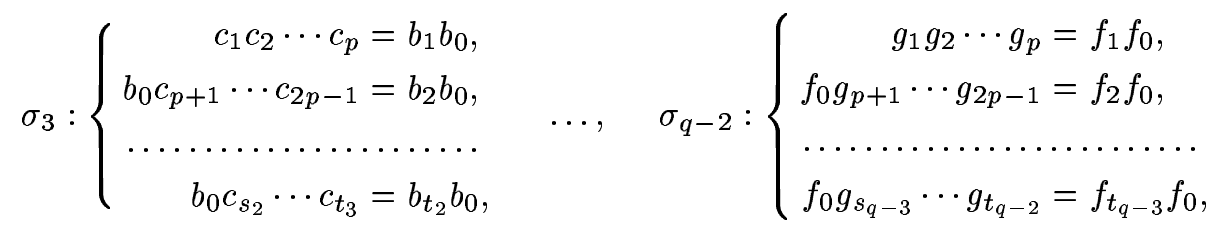

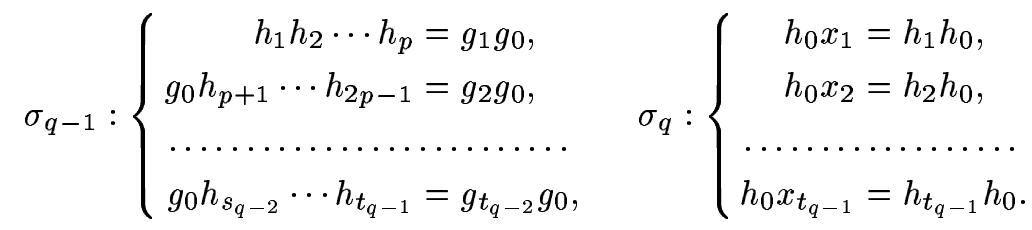

Заметим, что все образуюшие полугрупшы $S$, кроме $x_{0}, x_{1}, \ldots, x_{t}$, являются кусками относительно $\sigma$. Далее, нет кусков длины больше 1. Отсюда следует, что левые части равенств систем $\sigma_{1}, \sigma_{2}, \ldots, \sigma_{q-1}$ являются произведениями ровно $p$ кусков, а правые части равенств систем $\sigma_{2}, \ldots, \sigma_{q}$ состоят ровно из двух кусков. Правые части равенства $\sigma_{1}$ и левые части равенств $\sigma_{q}$ не разложимы в произведение кусков.

Проверим условие $D(q)$. Правьй граф полугрупшы $S$ не имеет циклов. Левьй же граф обладает единственньм циклом, определяемьп первьми соотношениями систем $\sigma_{1}, \sigma_{2}, \ldots, \sigma_{q}$. Этот цикл с $q$ ребрами:

$$
\left(h_{0}, a_{1}\right),\left(a_{1}, b_{1}\right),\left(b_{1}, c_{1}\right), \ldots,\left(f_{1}, g_{1}\right),\left(g_{1}, h_{1}\right),\left(h_{1}, h_{0}\right) .
$$


Остается показать, что в $S$ имеет место случай невыполнения сокращения слева. Следствиями соотношений $\sigma$ являются следуюшие равенства:

$$
\begin{gathered}
h_{0} x_{0}=a_{1} \cdots a_{t_{1}}, \quad a_{1} \cdots a_{t_{1}} a_{0}=b_{1} \cdots b_{t_{2}} \\
b_{1} \cdots b_{t_{2}} b_{0}=c_{1} \cdots c_{t_{3}}, \quad \cdots, \quad f_{1} \cdots f_{t_{q-3}} f_{0}=g_{1} \cdots g_{t_{q-2}} \\
g_{1} \cdots g_{t_{q-2}} g_{0}=h_{1} \cdots h_{t_{q-1}}, \quad h_{1} \cdots h_{t_{q-1}} h_{0}=h_{0} x_{1} \cdots x_{t_{q-1}} .
\end{gathered}
$$

Отсюда вытекает, что

$$
h_{0} x_{0} a_{0} b_{0} a_{0} \cdots f_{0} g_{0} h_{0}=h_{0} x_{1} \cdots x_{t_{q-1}} .
$$

При сокращении слева на $h_{0}$ получим равенство двух графически не совпадающих слов, каждое из которых не содержит вхождений определяющих слов полугрупшы $S$, что не может иметь места в $S$. Теорема доказана.

ПримеР. Рассмотрим следующую полугрупу $S$ из класса $K_{2, \infty}^{2}$ :

$$
S=\left\langle\left\langle a, b, c, x, y, z ; a^{2}=c x, a b=c y, b^{2}=a z\right\rangle\right\rangle
$$

Очевидно, $c x z=a^{2} z=a b^{2}=c y b$ в $S$. Поскольку $x z \neq y b$ в $S$, то в $S$ не вьполняется сокращение слева и, следовательно, $S$ не вложима в группу.

ЗАмЕчАниЕ. Правые графы полугрупп, построенных в теореме 2 и в только что рассмотренном примере, не содержат циклов, поэтому (см. [2, теорема 1]) в этих полугруппах выполняется правостороннее сокращение. Вопрос о вложимости в группу полугрупп с двусторонним сокращением из классов $K_{2, p}^{q}(p \geqslant 3, q \geqslant 2)$ и $K_{2, \infty}^{2}$ остается открытым.

В оставшейся части заметки мы рассмотрим некоторый класс полугрупп $\left(K_{2}^{2}\right)^{\Xi}$, включающий класс $K_{2, \infty}^{3}$, и докажем (теорема 3 ), что все полугрупшы из этого класса вложимы в группу.

Пусть $\Gamma$ - левый граф в смысле Адяна задания (1) полугруппы $S$. Построим новый граф $\Gamma^{*}$, заменяя некоторые ребра графа $\Gamma$ на ориентированные. Пусть $A=B$-определяющее соотношение полугрупшы $S, A \equiv a A^{\prime}, B \equiv b B^{\prime}$ и $e$ - соответствующее ребро графа $\Gamma$, соединяющее буквы $a$ и $b$. Букву $x$ назовем изолированной, если $x$ входит не более одного раза в определяющие соотношения полугрупш $S$ (и, следовательно, не может входить ни в какой $S$-кусок). В зависимости от того, содержат или нет слова $A$ и $B$ изолированные буквы, рассмотрим четыре случая:

1) если $A$ и $B$ не содержат изолированных букв, то ребро е оставляем неориентированным;

2 ) если $A$ содержит изолированную букву, а $B$ - нет, то ребро $e$ ориентируем от $b$ к $a$;

3) если $A$ не содержит изолированньх букв, а $B$ содержит изолированную букву, то $e$ ориентируем от $a \mathrm{~K} b$;

4 ) если оба слова $A$ и $B$ содержат изолированные буквы, то ребро $e$ считаем ориентированньм одновременно в обоих возможных направлениях.

Аналогично строится правьй граф $\Gamma^{*}$ для $S$. 


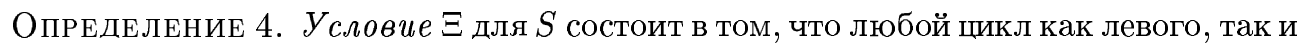
правого графа $\Gamma^{*}$ для $S$ содержит по меньшей мере два ребра, ориентированных в одном направлении. По определению класс $\left(K_{2}^{2}\right) \Xi$ состоит из всех полугруп, удовлетворяющих условиям $C_{s}(2), D(2)$ и $\Xi$.

ЗАмЕчАниЕ. Для вьполнения условия $\Xi$ достаточно наличия в каждом цикле трех ориентированных ребер. Условие $C_{s}(2, \infty)$ гарантирует ориентированность каждого ребра графа $\Gamma^{*}$. Отсюда следует $K_{2, \infty}^{3} \subset\left(K_{2}^{2}\right)^{\Xi}$.

Теорема 3. Любая полугруппа $S$ из класса $\left(K_{2}^{2}\right)^{\Xi}$ вложима в группу.

ДоКАЗАТЕЛЬСТво. Пусть $M$ - связная односвязная диаграмма над заданием группы $G$. Будем рассматривать $M$ и как ориентированный граф в обычном смысле. Для этого каждое ребро диаграммы $M$ разбиваем, насколько это необходимо, вершинами степени 2 на ребра, имеющие только позитивные или негативные метки. Каждому ребру е полученной диаграммы $M^{\prime}$ приписываем ориентацию, в направлении которой его метка $\varphi(e)$ читается как позитивное слово. Такую диаграмму $M^{\prime}$ будем называть $n о з и-$ тивно ориентированной. Вершину $v$ позитивно ориентированной диаграммы такую, что $v$ не имеет входящих в нее ребер, будем называть источником. Если $v$ не имеет выходящих из нее ребер, то $v$ назовем стоком. Если при этом $v$ - внутренняя вершина, то $v$ называем соответственно внутренним источником и внутренним стоком.

Пусть $M$ - позитивно ориентированная диаграмма над заданием (2) групы $G$ с теми же соотношениями, что и у полугрупы $S$. Предположим, что метка граничного контура $M$ имеет вид $U V^{-1}$, где $U$ и $V$ - позитивные слова. Реммерс [9, теорема 3.2] показал, что если $M$ не имеет ни внутренних источников, ни внутренних стоков, то $U \stackrel{s}{=} V$.

Таким образом, для доказательства вложимости полугрупшы $S$ в групш $G$ достаточно показать, что для любьх позитивньх слов $U, V$ из $U \stackrel{g}{=} V$ вытекает существование позитивно ориентированной диаграммы $M$ над заданием (2) групшы $G$ с меткой граничного цикла $U V^{-1}$, не содержащей внутренних источников и стоков.

Пусть $S \in\left(K_{2}^{2}\right)^{\Xi}$, и пусть $U$ и $V$ - позитивные слова такие, что $U \stackrel{g}{=} V$. Пусть $M-$ приведенная позитивно ориентированная диаграмма над (2) с граничным циклом $\nu \tau^{-1}$, где $\varphi(\nu) \equiv U$ и $\varphi(\tau) \equiv V$. Индукцией по числу $d(M)$ областей диаграммы $M$ докажем, что $M$ не содержит внутренних источников и стоков.

Если $d(M) \leqslant 1$, то утверждение очевидно. Пусть $d(M)>1$. Допустим, что вершина $v$ диаграммы $M$ является внутренним источником или внутренним стоком. Предположим для определенности, что $v$-внутренний источник (для случая, когда $v$-внутренний сток, рассуждения симметричны). Пусть $T_{1}, T_{2}, \ldots, T_{m}$ - метки всех ребер, выходящих из $v$ и пронумерованных в положительном направлении обхода вокруг вершины $v$. Так как $T_{i}$ - позитивные слова, то для некоторых позитивных слов $U_{i}, V_{i}(i=1,2, \ldots, m)$ слова

$$
T_{1} U_{1} V_{1}^{-1} T_{2}^{-1}, T_{2} U_{2} V_{2}^{-1} T_{3}^{-1}, \ldots, T_{m-1} U_{m-1} V_{m-1}^{-1} T_{m}^{-1}, T_{m} U_{m} V_{m}^{-1} T_{1}^{-1}
$$

являются метками областей, границе которых принадлежит вершина $v$. Тогда соотношения

$$
T_{1} U_{1}=T_{2} V_{1}, \quad T_{2} U_{2}=T_{3} V_{2}, \ldots, T_{m-1} U_{m-1}=T_{m} V_{m-1}, \quad T_{m} U_{m}=T_{1} V_{m}
$$


с точностью до перестановки левьх и правьх частей являются определяющими соотношениями полугруппы $S$.

Из неприводимости диаграммы $M$ следует, что метки внутренних ребер являются кусками. Следовательно, $T_{1}, T_{2}, \ldots, T_{m}$ - куски и по лемме $1-S$-куски. Каждое из слов $U_{i}$ непусто, в противном случае $T_{i}$ является одновременно определяющим словом и куском, что невозможно по условию $C_{s}(2)$. По той же причине непусты слова $V_{i}$.

По условию $\Xi$ либо среди левых, либо среди правьх частей соотношений (10) найдутся по меньшей мере два слова, содержашие изолированные буквы. Рассмотрим случай, когда такими словами являются левые части $A_{j} \equiv\left(T_{j} U_{j}\right)^{*}$ и $A_{k} \equiv\left(T_{k} U_{k}\right)^{*}(j<k)$. Пусть $\alpha$ и $\beta$ - пути с метками $\varphi(\alpha) \equiv A_{j}, \varphi(\beta) \equiv A_{k}$. Так как изолированные буквы не могут входить в куски, то каждьй из путей $\alpha$ и $\beta$ содержит граничное ребро. Пусть $\alpha=\alpha_{1} \alpha_{2} \alpha_{3}$ и $\beta=\beta_{1} \beta_{2} \beta_{3}$, где $\alpha_{2}$ и $\beta_{2}$ - некоторые максимальные подпути путей $\alpha$ и $\beta$, состоящие только из граничньх ребер диаграммы $M$, причем, можно считать, что метка пути $\alpha_{2}$ содержит изолированную букву, а путь $\beta_{1}$ не содержит граничных ребер. Учитывая, что граничные циклы областей и всей диаграммы $M$ обходятся в одном направлении, получаем, что $\alpha_{2}$ и $\beta_{2}$ входят в $\nu$. Пусть $\gamma$ - подпуть пути $\nu$, начало которого совпадает с концом $\alpha_{2}$, а конец - с начальной вершиной $\beta_{2}$ (рис. 2 ).

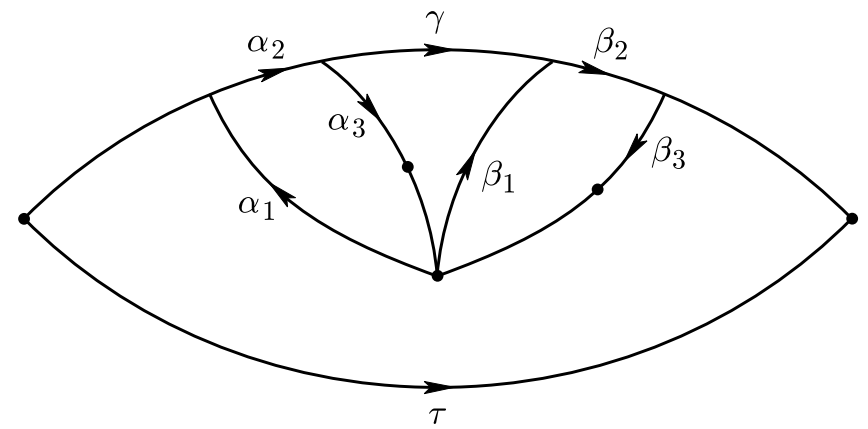

Рис. 2

Пусть $M_{1}$ - поддиаграмма диаграммы $M$ с граничньм контуром $\alpha_{1} \alpha_{2} \gamma \beta_{1}^{-1}$. Пусть $\varphi\left(\alpha_{i}\right)=X_{i}(i=1,2), \varphi\left(\beta_{1}\right)=Y_{1}$ и $\varphi(\gamma)=Z$. Тогда $X_{1} X_{2} Z \stackrel{g}{=} Y_{1}$. Поскольку $d\left(M_{1}\right)<d(M)$, то по индуктивному предположению $X_{1} X_{2} Z \stackrel{s}{=} Y_{1}$. Поскольку $Y_{1}-$ собственное начало $A_{k}$, то в силу условия $C_{s}(2)$ к нему неприменимо никакое соотношение полугруппы $S$. Следовательно, $X_{1} X_{2} Z \equiv Y_{1}$. Но это невозможно, так как слово $Y_{1}$ как метка внутреннего пути $\beta_{1}$ разложимо в произведение кусков, а $X_{2} Z$ - нет, поскольку метка пути $\alpha_{2} \gamma$ содержит изолированную букву.

Случай, когда слова, содержащие изолированные буквы, находятся среди правых частей соотношений (10), симметричен рассмотренному. Таким образом, мы получили противоречие из предположения о существовании в $M$ внутреннего источника. В случае, когда $M$ обладает внутренним стоком, рассуждения совершенно аналогичны. Теорема 3 доказана. 
ПРИМЕРЫ. Полугруппы

$$
\begin{gathered}
\langle\langle a, b, c, d, x, y, z ; a b=b x c, b a=c y c, c a=a z b\rangle\rangle, \\
\left\langle\left\langle a, b, c, x, y, z ; c^{2}=a x, a b=c y, b^{2}=a z\right\rangle\right\rangle
\end{gathered}
$$

лежат в классе $\left(K_{2}^{2}\right)^{\Xi}$ и по теореме 3 вложимы в группу. Первая принадлежит классу $K_{2, \infty}^{3}$, а вторая не принадлежит.

\section{СПИСОК ЦИТИРОВАННОЙ ЛИТЕРАТУРЫ}

[1] Адян С. И. О вложимости полугрупп в группы // Докл. АН СССР. 1960. Т. 133. № 2. C. $255-257$.

[2] Адян С. И. Определяюшие соотношения и алгоритмические проблемы для групп и полугрупп. Тр. МИАН. Т. 85. М.: Наука, 1966.

[3] Kashintsev E. V. Small cancellation conditions and embeddability of semigroups in groups // Intern. J. Algebra Comput. 1992. V. 2. № 4. P. 433-441.

[4] Maltsev A. I. On the immersion of an algebraic ring into a field // Math. Ann. 1937. V. 133. P. 689-691.

[5] Губа В. С. Об условиях вложимости полугрупп в группы // Матем. заметки. 1994. Т. 56. №2. C. $3-14$.

[6] Kilgour C. W. Embedding Monoids in Groups. Preprint: Univ. of Glasgow, 1997.

[7] Линдон Р., Шупп П. Комбинаторная теория групп. М.: Мир, 1980.

[8] Cho J. R., Pride S. J. Embedding semigroups into groups and the asphericity of semigroups // Intern. J. Algebra Comput. 1993. V. 3. № 1. P. 1-13.

[9] Remmers J. H. On the geometry of semigroup presentations // Adv. Math. 1980. V. 36. P. 283-296. 